\title{
Robust Switched Predictive Braking Control for Rollover Prevention in Wheeled Vehicles
}

\author{
Martín Antonio Rodríguez Licea and Ilse Cervantes \\ Hybrid Systems Laboratory, Applied Mathematics Division, Institute for Scientific and Technological Research of \\ San Luis Potosi (IPICyT), Camino a la Presa San Jose 2055, Col. Lomas 4ta Seccion, 78216 San Luis Potosi, SLP, Mexico \\ Correspondence should be addressed to Martín Antonio Rodríguez Licea; martin_antonio_r@hotmail.com
}

Received 29 December 2013; Accepted 24 March 2014; Published 24 April 2014

Academic Editor: Sheldon S. Williamson

Copyright (C) 2014 M. A. R. Licea and I. Cervantes. This is an open access article distributed under the Creative Commons Attribution License, which permits unrestricted use, distribution, and reproduction in any medium, provided the original work is properly cited.

\begin{abstract}
The aim of this paper is to propose a differential braking rollover mitigation strategy for wheeled vehicles. The strategy makes use of a polytopic (piecewise linear) description of the vehicle and includes translational and rotational dynamics, as well as suspension effects. The braking controller is robust and the system states are predicted to estimate the rollover risk up to a given time horizon. In contrast to existing works, the switched predictive nature of the control allows it to be applied only when risk of rollover is foreseen, interfering a minimum with driver's actions. The stability of the strategy is analyzed and its robustness is illustrated via numerical simulations using CarSim for a variety of vehicles.
\end{abstract}

\section{Introduction}

Vehicle rollover is a serious problem that commonly involves sport utility vehicles (SUVs), trailers, trucks, and, in general, vehicles with a high center of gravity $(\mathrm{CoG})$. Many quantifications of rollover risk exist in the literature [1-5]. The most commonly used is the load transfer ratio (LTR), also known as rollover index (RI) proposed by National Highway Traffic Safety Administration (NAFTA) [1], which is an index that measures the balance of vertical forces at the tire-road contact points:

$$
\mathrm{RI}=\mathrm{LTR}=\frac{\sum F_{\mathrm{ZL}}-\sum F_{\mathrm{ZR}}}{\sum F_{\mathrm{ZL}}+\sum F_{\mathrm{ZR}}},
$$

where $F_{Z L}, F_{Z R}$ are the sum of left and right side tireroad vertical forces, respectively. RI is dimensionless and constitutes a balance between right and left vertical forces, with $\mathrm{RI}= \pm 1$ indicating imminent rollover (the whole weight of the vehicle is on its left or right side) and $\mathrm{RI}=0$ indicating a perfect balanced vehicle. The RI is used to describe rollover risk in any four-wheeled vehicle since it is related to a balance of vertical forces. $|\mathrm{RI}|$ values below 0.6 are considered safe [1]. Since RI is a force balance, it is directly related to the car lateral inclination and it can be measured as a function of chassis roll angle $(\varphi)$ and chassis roll angle velocity $(\dot{\varphi})$. Although $\varphi, \dot{\varphi}$ can be measured it is not the case of the tire-road contact point forces. The estimation of these forces is complex to obtain since it depends nonlinearly on the vehicle's roll angle and speed and also on the type of terrain, tires, suspension, driver behavior, type of vehicle, and so forth; accordingly, there are several works in the literature dealing with the estimation of these forces [5-8].

Unfortunately, the RI estimation and a detonation of a warning of rollover cannot be used alone to mitigate the rollover phenomenon since human reaction delay and driver experience are limiting factors. Rollover can be avoided only by using effective timely actions on either active suspension, active steering, active differential, or differential braking [913]. Among the aforementioned actuators, perhaps the differential braking strategy is the easiest to implement since only antilock braking system (ABS) is necessary, contrasting to the other actuators which require costly and/or nonstandard devices. Since the aim of this work is to propose a differential braking control strategy, the literature review will be focused on these works only.

There exist in the literature works dealing with robust, switched, and/or predictive differential braking controllers. In particular, robustness of a control is a desired property 
since nonlinearities of the dynamics, particularly those of tires and suspension, are hard to describe. Furthermore, exhaustive nonlinear models cannot be used for control design purposes since the problem becomes hardly tractable. Instead, approximated (linear) models are used to describe the vehicle dynamics and to simplify control synthesis. Since a single linear model cannot reproduce the system's complexity, polytopic or piecewise linear models are better options that guarantee tractability of the control design and of the stability analysis problem [14-16].

Robust differential braking controllers are proposed in [17-19]. In [17], a parametric uncertainty robust control strategy is proposed using a linear description of the vehicle including the suspension dynamics, leading to control actions that are applied continuously at all times. In [18], a robust control design based on linear matrix inequalities (LMIs) is proposed. The control is based on a vehicle description that uses the speed as uncertain parameter. The controller is shown to be robust to parameter uncertainty, but it is not clear if it can deal with the uncertainty in the whole operation domain and with the nonlinearities of the system. An adaptive switching controller is proposed in [19]. Least squares and Kalman filtering techniques are employed to estimate the height of the center of gravity in face of speed variations.

Since timely control actions depend on driver's decisions as well as on system and actuator inner dynamics, more recent works use the prediction of system states to compensate for such dynamics. In [20] a predictive RI strategy based on the present values of RI and steering angle is proposed. The resulting controller displays good performance avoiding rollover; however, the control action is applied at all times, interfering adversely with the driver's commands, even when no rollover risk is foreseen. Other works focusing on predictive and robust control actions are [21, 22]. In [21] a nonlinear inverted pendulum-like vehicle model is used to design a model predictive controller that is able to be applied in real-time. Even if the controller is not shown to be robust, the contribution of the authors is to take into account some of the nonlinearities of the system. In [22] a back-stepping observer is designed to estimate a lateral sliding and rollover indicator online. A maximum velocity tracking is obtained using predictive functional control.

Although these works constitute important advances, either they interfere with the drivers commands expending control actions even when no imminent risk of rollover is detected, or the robustness of the control in face of nonlinearities is not clear.

In this work we are focused on developing a noninvasive braking control that allows the driver to manipulate freely the vehicle until a rollover risk is foreseen. The controller is predictive and robust and guarantees stability in face of uncertainties using a polytopic linear model that takes into account different behaviors at a variety of operation conditions. The control is switched and its on-off control actions are performed using simple time/state dependent criteria based on the prediction. These three features: robustness, prediction, and switching make the controller versatile and its structure and proof of stability constitute the main contribution of this paper.

The prediction of the states is obtained using a set of linear models and an estimation of the future steering input. The steering prediction is obtained using a zero-order model of the driver. The predicted vehicle dynamics are used to estimate the RI behavior up to the time horizon in order to determine whether or not a rollover risk exists. If such possibility is present, a switched controller is used to mitigate the rollover phenomenon. The controller has a supervisory structure and it is comprised of a set of time- and statedependent switching rules and a feedback braking control action.

The effect of the prediction horizon on system performance, as measured with the RI, is studied and compared against another nonswitched scheme. The proposed strategy has the feature of being simple for implementation purposes and it uses ABS braking technology. Moreover, it does not make use of GPS for driving prediction.

This document is organized as follows: in Section 2 the vehicle model is derived. Sections 3 and 4 introduce, respectively, the problem statement and the main contribution of this paper. The performance of the proposed controller is illustrated using numerical simulations in Section 5. Finally, in Section 6 some conclusions are presented.

\section{Vehicle Model}

Consider the nonlinear model of the vehicle given by

$$
\begin{gathered}
\dot{v}_{\mathrm{CoG}}=\frac{\sin (\beta)}{m}\left(\sum F_{\mathrm{Sij}}+\left(F_{\mathrm{LFL}}+F_{\mathrm{LFR}}\right) \delta+F_{\mathrm{YT}}\right) \\
+\frac{\cos (\beta)}{m}\left(\sum F_{\mathrm{Lij}}-\left(F_{\mathrm{SFL}}+F_{\mathrm{SFR}}\right) \delta+F_{\mathrm{XT}}\right), \\
\dot{\beta}=\frac{\cos (\beta)}{m v_{\mathrm{CoG}}}\left(\sum F_{\mathrm{Sij}}+\left(F_{\mathrm{LFL}}+F_{\mathrm{LFR}}\right) \delta+F_{\mathrm{YT}}\right) \\
\quad-\frac{\sin (\beta)}{m v_{\mathrm{CoG}}}\left(\sum F_{\mathrm{Lij}}-\left(F_{\mathrm{SFL}}+F_{\mathrm{SFR}}\right) \delta+F_{\mathrm{XT}}\right), \\
\ddot{\psi}=\left(F_{\mathrm{SFR}}+F_{\mathrm{SFL}}+\left(F_{\mathrm{LFL}}+F_{\mathrm{LFR}}\right) \delta\right) \frac{L_{v}}{J_{Z}}-\left(F_{\mathrm{SRR}}+F_{\mathrm{SRL}}\right) \frac{L_{h}}{J_{Z}} \\
+\left(F_{\mathrm{LRR}}+F_{\mathrm{LFR}}-F_{\mathrm{LFL}}-F_{\mathrm{LRL}}+\left(F_{\mathrm{SFL}}-F_{\mathrm{SFR}}\right) \delta\right) \frac{b}{2 J_{Z}}, \\
\ddot{\varphi}=\frac{1}{J_{x}}\left(h_{\mathrm{CoG}}\left(\sum F_{\mathrm{Sij}}+\left(F_{\mathrm{LFL}}+F_{\mathrm{LFR}}\right) \delta+F_{\mathrm{YT}}\right)-k \varphi-c \dot{\varphi}\right),
\end{gathered}
$$

where $F_{\mathrm{Sij}}$ are the lateral tire forces, $F_{\mathrm{Lij}}$ are the braking/acceleration forces with $i=\{$ Front, Rear $\}, j=\{$ Left, Right $\}, \delta$ is the front wheels angle, and $F_{\mathrm{XT}}, F_{\mathrm{YT}}$ are terms that account for the gravity, rolling resistance, and other forces. $L_{v}$, $L_{h}, J_{z}$, and $h$ are suitable constants. See Nomenclature section. 
Forces $F_{\mathrm{Sij}}$ are highly nonlinear and dependent on the front and rear tire side slip angles $\left(\alpha_{v}, \alpha_{h}\right)$, as well as on road and tire parameters $[23,24]$. Let

$$
\begin{gathered}
\alpha_{v}=\delta-\beta-\frac{L_{v} \dot{\psi}}{v_{\mathrm{CoG}},} \\
\alpha_{h}=-\beta+\frac{L_{h} \dot{\psi}}{v_{\mathrm{CoG}}}, \\
F_{\mathrm{LFL}}+F_{\mathrm{LRL}}= \begin{cases}u, & u<0, \\
0, & u \geq 0,\end{cases} \\
F_{\mathrm{LFR}}+F_{\mathrm{LRR}}= \begin{cases}-u, & u>0, \\
0, & u \leq 0 .\end{cases}
\end{gathered}
$$

Let $x^{\prime}=[\beta \dot{\psi} \dot{\varphi} \varphi]$ (where $x^{\prime}$ denotes the transpose of $x$ ); the linearized vehicle dynamics about a speed dependent operation point becomes

$$
\dot{x}=A x+B_{\delta} \delta(t)+B_{u} u,
$$

with

$$
\begin{gathered}
A=\left[\begin{array}{cccc}
-\frac{\sigma J_{x_{\mathrm{eq}}}}{m \widehat{v}_{\mathrm{CoG}} J_{x}} & \frac{\rho J_{x_{\mathrm{eq}}}}{m \widehat{v}_{\mathrm{CoG}}^{2} J_{x}}-1-\frac{h c}{J_{x} \widehat{v}_{\mathrm{CoG}}} & \frac{h(m g h-k)}{J_{x} \widehat{v}_{\mathrm{COG}}} \\
\frac{\rho}{J_{z}} & -\frac{\kappa}{J_{z} \widehat{v}_{\mathrm{CoG}}} & 0 & 0 \\
-\frac{h \sigma}{J_{x}} & \frac{h \rho}{\widehat{v}_{\mathrm{CoG}} J_{x}} & -\frac{c}{J_{x}} & \frac{m g h-k}{J_{x}} \\
0 & 0 & 1 & 0
\end{array}\right], \\
B_{\delta}=\left[\begin{array}{ccc}
\frac{c_{v} J_{x_{\mathrm{eq}}}}{m J_{x} \widehat{v}_{\mathrm{CoG}}} \frac{c_{v} L_{v}}{J_{z}} & \frac{h c_{v}}{J_{x}} & 0
\end{array}\right]^{\prime}, \\
B_{u}=\left[\begin{array}{lll}
0-\frac{b}{2 J_{z}} & 0 & 0
\end{array}\right]^{\prime}, \\
\sigma \triangleq c_{v}+c_{h}, \\
\rho \triangleq c_{h} L_{h}-c_{v} L_{v}, \\
\kappa \triangleq c_{h} L_{h}^{2}+c_{v} L_{v}^{2}, \\
J_{x_{\mathrm{eq}}} \triangleq J_{x}+m h^{2},
\end{gathered}
$$

where $\widehat{v}_{\mathrm{COG}}$ is the nominal velocity value and $u$ represents the differential braking force. Using this model, the RI is given by (see [25])

$$
\mathrm{RI}(t)=-\frac{2}{b m g}(c \dot{\varphi}+k \varphi)
$$

2.1. Nonlinearities and Parametric Uncertainty. The linear model (4)-(5) has been derived as an approximation of the nonlinear dynamics (2), about a given operation point. In

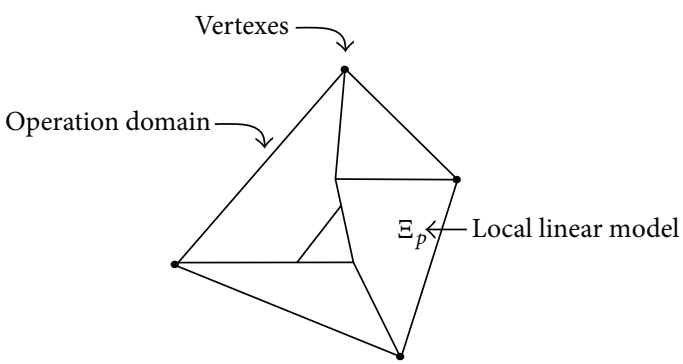

Figure 1: Schematic diagram of the operation domain $\mathscr{D}$. The domain is divided into finite sets $\Xi_{p}$ and, associated with each one, there is a linear approximation of the system. The description of the system inside $\mathscr{D}$ can be performed using a convex combination of the parameters of the models.

the following, we will derive a polytopic linear model of the system such that not only small deviations of the operation point are allowed to describe the system dynamics, but also large ones, as long as they occur in a closed operation domain. To this end, assume that the operation domain $\mathscr{D}$ is divided into a finite number of sets $\Xi_{p}$ for $p=1,2, \ldots$, nd, each one linked to a unique linear model expression (see Figure 1). Sets $\Xi_{p}$ satisfy

$$
\begin{aligned}
& \text { (1) } \bigcup_{p=1}^{\text {nd }} \Xi_{p}=\mathscr{D}, \\
& \text { (2) } \operatorname{Int}\left(\Xi_{p}\right) \bigcap \operatorname{Int}\left(\Xi_{l}\right)=\emptyset \text { for } p \neq l \text {, } \\
& \text { (3) } \Xi_{p} \neq \emptyset,
\end{aligned}
$$

where Int stands for the interior of the set. If the description of the system inside $\mathscr{D}$ can be performed using a convex combination of the parameters of every linear model associated with $\Xi_{p}$ then a robust continuous control can be derived to guarantee the stability in $\mathscr{D}[26]$. The convex parameter combination leads to a so-called polytopic model. In order to describe such a model, let us introduce the following definitions.

Definition 1. The set

$$
\Theta=\left\{\theta \in R \mid \sum_{i=1}^{\mathrm{nv}} \theta_{i}=1, \theta_{i} \geq 0\right\}
$$

is called simplex and nv is called the number of vertexes.

Definition 2. The matrix $A$ is called polytopic if

$$
A=\left\{A(\theta) \mid \sum_{i=1}^{\mathrm{nv}} A_{i} \theta_{i}=A, \theta_{i} \geq 0\right\} .
$$

Using the notations above, the model of the vehicle can be rewritten as

$$
\dot{x}=A(\theta) x+B_{\delta}(\theta) \delta+B_{u}(\theta) u,
$$

where $A(\theta), B_{\delta}(\theta)$, and $B_{u}(\theta)$ are polytopic matrices of suitable dimensions and $\delta(t): \mathbb{R}^{+} \rightarrow \mathbb{R}$, the front wheels angle generated by the steering input, is a piecewise continuous 
function of time. According to Definitions 1 and 2, the parameters in the polytopic matrices vary within closed and bounded ranges, where each extremum is characterized by the simplex vertexes $\theta_{i}=1$. As a result, it is possible to divide the domain $\mathscr{D}$ in an arbitrary finite number of subsets $\Xi_{p}$ (see Figure 1). In other words, model (10) is actually a multilinear model that can be constructed using a finite number of linear approximations (4) at different operating conditions (e.g., vehicle speed, tire pressure, type of terrain, etc.). In Section 5 we illustrate the derivation of the multilinear model for a Cherokee Jeep 2000.

\section{Controller Structure}

In the literature, the differential braking control problem has been stated as follows.

Problem 3. Consider the vehicle model (10) and assume that the state $x$ is available for measurement; design a control law $u(t)$ such that

$$
|\mathrm{RI}(t)| \leq \mathrm{RI}_{\mathrm{ref}},
$$

for all time $t$ and all parameters in the simplex $\Theta$, where $\mathrm{RI}_{\text {ref }}$ stands for a reasonable value of RI such that the vehicle has no rollover risk.

The control objective (11) can be attained using robust control theory if the pair $\left(A(\theta), B_{u}(\theta)\right)$ is completely controllable and if it is possible to solve linear matrix inequalities (LMIs) associated with a Lyapunov equation [26]. In this case, a suitable vector $K$ can be found, such that the control law

$$
u(t)=K x,
$$

makes system (10) $L_{\infty}$ stable for all $|\delta| \leq \delta_{s}$, where $\delta_{s}$ stands for the maximum magnitude of the front wheels angle to avoid violating the restriction $|\mathrm{RI}(t)| \leq \mathrm{RI}_{\text {ref }}$. By solving the LMI it is ensured that $A_{2}(\theta)=A(\theta)+B_{u}(\theta) K$ is Hurwitz in the simplex $\Theta$.

This approach has been discussed in the past, for a simpler model in $[17,25]$. However, the said approach has the disadvantage of being continuously applied in the vehicle, consuming braking force and interfering constantly with the velocity path set by the driver, even when no risk of rollover exists. To avoid such disadvantages the problem addressed in this paper can be formulated as follows.

Problem 4. Consider the vehicle model (10) with $x$ available for measurement; design a differential braking control that guarantees

$$
|\mathrm{RI}(t)| \leq \mathrm{RI}_{\mathrm{ref}},
$$

for all time $t$ and all parameters in the simplex $\Theta$, such that the controller is applied only when a predicted value of RI satisfies $\mathrm{RI}>\mathrm{RI}_{\text {ref }}$.

To solve Problem 4, a two-mode switched, noninvasive approach is proposed in this work. The first mode (Mode 1) coincides with the vehicle dynamics as conducted by the driver (open-loop), while the other one (Mode 2) applies the robust control (12) when necessary to attain the control objective (13).

If the Mode 2 is activated once the boundary $|\mathrm{RI}(t)|=$ $\mathrm{RI}_{\text {ref }}$ is reached, there is no guarantee of the accomplishment of (13). An alternate solution would be to set a lower bound of $\mathrm{RI}(t)$ to compensate for system dynamics. In this work, we use a prediction of the state vector up to a time horizon $(T)$ to switch to Mode 2. If a rollover risk is detected within the time interval $[t, t+T]$, then Mode 2 is activated (i.e., $\mathrm{RI}\left(t_{T}\right) \leq \mathrm{RI}_{\text {ref }}$ with $\left.t_{T} \in[t, t+T]\right)$.

The rollover risk prediction is a tool that allows us to take corrective actions before the risk is imminent, and as the prediction horizon $T$ becomes larger, it is expected that the control becomes more invasive. The operation modes of the proposed methodology are described below with the help of a switching law:

$$
\begin{gathered}
\dot{x}=A_{i}(\theta) x+B_{\delta}(\theta) \delta, \\
i= \begin{cases}1, & \text { if }\left|\mathrm{RI}\left(t_{T}\right)\right| \leq \mathrm{RI}_{\mathrm{ref}} \wedge t_{r}>0(\text { Mode } 1), \\
2, & \text { if }\left|\mathrm{RI}\left(t_{T}\right)\right|>\mathrm{RI}_{\mathrm{ref}}(\text { Mode } 2), \\
& t_{T} \in[t, t+T], \quad T>0,\end{cases}
\end{gathered}
$$

where $A_{1}(\theta)=A(\theta), A_{2}(\theta)=A(\theta)+B_{u}(\theta) K$, and $t_{r}$ is a minimum residence time of Mode 2 . The block diagram of the proposed controller and the switching algorithm are shown in Figures 2 and 3, respectively.

To predict the behavior of the vehicle using the model (14), the prediction of the driver behavior is needed. Although modeling driving behavior constitutes a relevant and actual topic of research, it is out of the scope of this work. In this paper, we will assume that such model is available. For convenience, the approach in [27] will be used, with a zero order dynamics since it is simple and still adaptive to changing environments.

The proposed control works as follows. The driver provides the front wheels angle and the braking force through the steering wheel and the brake pedal, respectively. At every instant $t$, RI is compared with $\mathrm{RI}_{\text {ref }}$ along the prediction interval fixed by the prediction horizon. The RI estimation is performed using the vehicle model (14), (7), and the model of the driver behavior. If the threshold $\mathrm{RI}_{\text {ref }}$ is exceeded at some instant along the interval $[t, t+T]$, the Mode 2 is activated (i.e., a future rollover risk is detected); otherwise the Mode 1 is preserved. Once Mode 2 is activated, it continues being active until the rollover risk for the entire prediction horizon has disappeared and a minimum dwell time $t_{r}$ is elapsed. This dwell time prevents fast switching actions (chattering) improving the performance. The stability of the proposed control is studied in the following section, where stability properties of the switching actions are given.

\section{Main Result}

Let us denote the solution of (14) at time $t$ with initial condition $x_{0}=x\left(t_{0}\right)$, uncertain parameter within the simplex 


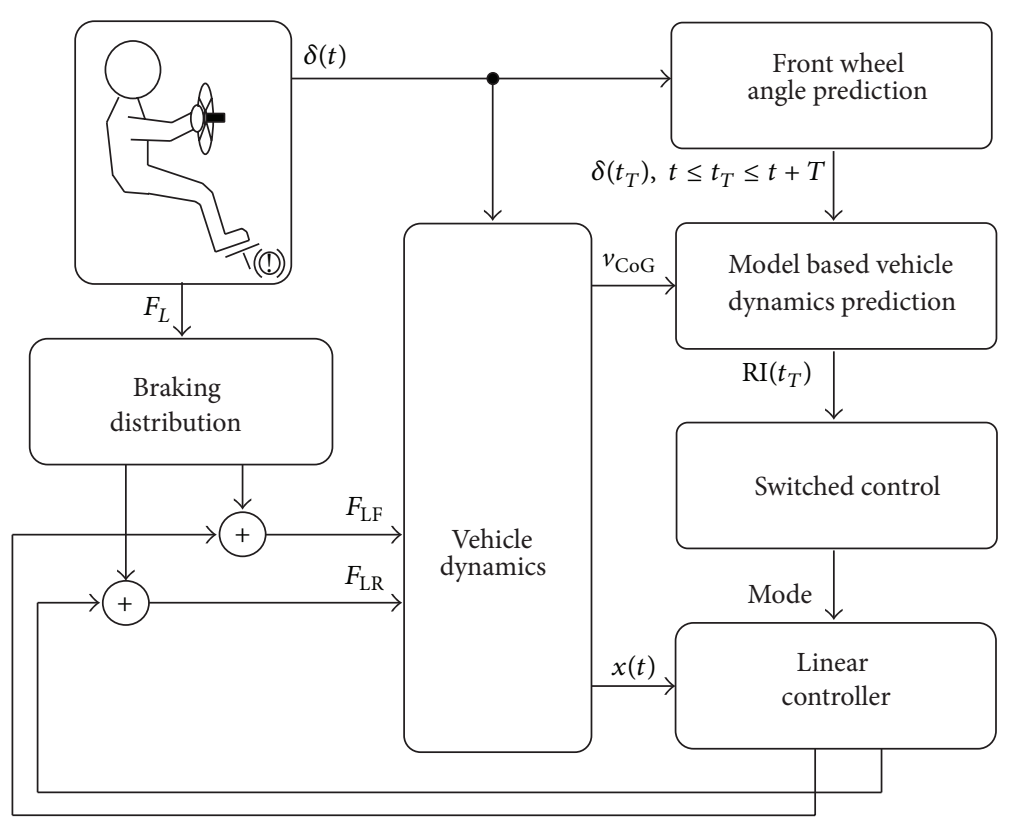

FIGURE 2: Block diagram of control strategy.

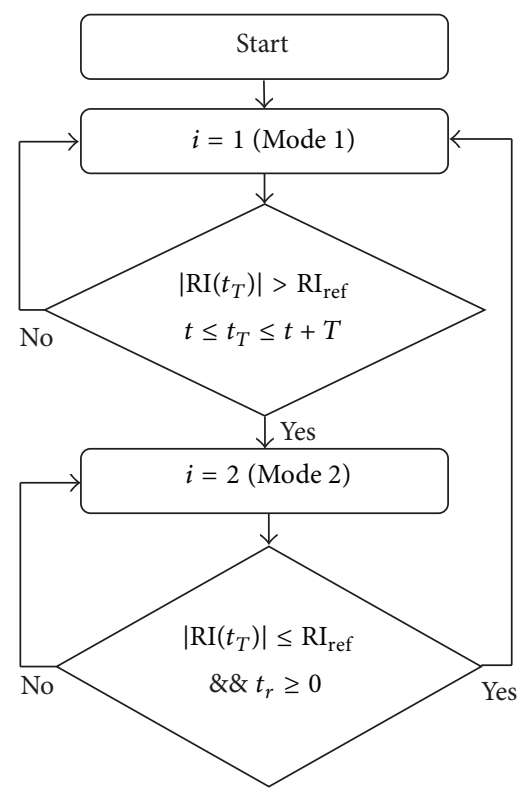

FIGURE 3: Algorithm of the switching criterion.

$\Theta$, and initial time $t_{0}$ as $x\left(t_{0}, t, x_{0}, \theta\right) . B_{\xi}(z)$ is the ball centered at $z$ with radius $\xi$ (i.e., $B_{\xi}(z)=\{x \mid\|x-z\| \leq \xi\}$ ).

For $i=1$, the unperturbed system (14) (i.e., $\delta=0$ ) has an open-loop equilibrium in $x^{\prime}=\left[\beta_{\mathrm{eq}}, \dot{\psi}_{\mathrm{eq}}, \dot{\varphi}_{\mathrm{eq}}, \varphi_{\mathrm{eq}}\right]=0$. By vehicle design, $A_{1}(\theta)$ is Hurwitz for all $\theta \in \Theta$; otherwise, the unperturbed vehicle would spin or rollover by following a straight line. On the other hand, the existence of a robust controller (12) such that $A_{2}(\theta)$ is also a Hurwitz matrix is guaranteed by the controllability of the pair $\left(A(\theta), B_{u}(\theta)\right)$.

The control objective (13) can be expressed from the stability point of view, as the property of positive invariance in some suitable set $(\Omega)$. The trajectories $x\left(t_{0}, t, x_{0}, \theta\right)$ are positively invariant in $\Omega$; that is, $x\left(t_{0}, t, x_{0}, \theta\right) \in \Omega$, for all $t \geq 0$.

Consider system (14) with initial conditions within $B_{\varepsilon}(0)$ with $\varepsilon>0$. Since for each mode $A_{i}$ is Hurwitz, then for each $\left\|x\left(t_{0}\right)\right\| \leq \varepsilon$ there exists a maximum value of $|\widehat{\delta}|_{\max }$ such that the system trajectories will remain within $B_{\rho}(0)$; that is, the system is input-output stable. The first step in deriving our main contribution is the quantification of such perturbation $|\widehat{\delta}|_{\max }$ under system commutation.

Problem 5. Consider the system

$$
\dot{x}=A_{i}(\theta) x+B_{\delta}(\theta) \delta,
$$

with $i=1,2$ and let

$$
\Omega \triangleq\left\{x\left(t_{0}, t, x_{0}, \theta\right)|| \mathrm{RI}(t) \mid \leq \mathrm{RI}_{\mathrm{ref}}\right\} .
$$

Establish switching conditions for the system (15) such that $x\left(t_{0}, t, x_{0}, \theta\right) \in \Omega$ for all $t \geq 0$.

Let $i=2$ and $t_{0}=0$ be the switching instant from Mode 1 to Mode 2. Then, the solution of (15) while evolving in Mode 2 is

$$
\begin{gathered}
x\left(0, t, x_{0}, \theta\right)=e^{A_{2}(\theta) t} x_{0}+\int_{0}^{t} e^{A_{2}(\theta)(t-\tau)} B_{\delta}(\theta) \delta(\tau) d \tau, \quad(17) \\
\mathrm{RI}(t)=C(\theta) e^{A_{2}(\theta) t} x_{0}+C(\theta)+\int_{0}^{t} e^{A_{2}(\theta)(t-\tau)} B_{\delta}(\theta) \delta(\tau) d \tau,
\end{gathered}
$$

with

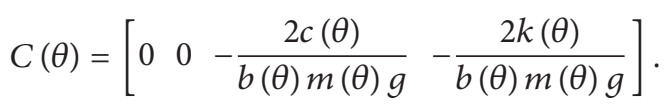


Computing the norm of (18) we have

$$
\begin{aligned}
\|\mathrm{RI}(t)\| \leq & \|C(\theta)\|\left\|e^{A_{2}(\theta) t}\right\|\left\|x_{0}\right\| \\
& +\|C(\theta)\|\left\|\left[I-e^{A_{2}(\theta) t}\right]\right\|\left\|A_{2}^{-1}(\theta) B_{\delta}(\theta)\right\||\delta| \\
\leq & \|C(\theta)\| \beta_{2}(\theta) e^{r_{2}(\theta) t} \varepsilon \\
& +\|C(\theta)\|\left[1+\beta_{2}(\theta) e^{r_{2}(\theta) t}\right]\left\|A_{2}^{-1}(\theta) B_{\delta}(\theta)\right\||\delta| \\
\leq & \|C(\theta)\| \beta_{2}(\theta) \varepsilon \\
& +\|C(\theta)\|\left[1+\beta_{2}(\theta)\right]\left\|A_{2}^{-1}(\theta) B_{\delta}(\theta)\right\||\delta| \leq \mathrm{RI}_{\mathrm{ref}},
\end{aligned}
$$

where Euclidean norm is denoted as $\|\cdot\|,\left\|x_{0}\right\| \leq \varepsilon$, and $\left\|e^{A_{2}(\theta) t}\right\| \leq \beta_{2}(\theta) e^{r_{2}(\theta) t}$ with $r_{2}(\theta)<0$.

From (20) the maximum perturbation that allows $x(0$, $\left.t, x_{0}, \theta\right) \in \Omega$ is

$$
|\delta|_{\max }<\pi(\theta, \varepsilon) \triangleq \frac{\mathrm{RI}_{\mathrm{ref}}-\|C(\theta)\| \beta_{2}(\theta) \varepsilon}{\|C(\theta)\|\left[1+\beta_{2}(\theta)\right]\left\|A_{2}^{-1}(\theta) B_{\delta}(\theta)\right\|} .
$$

Let

$$
\theta_{r 2}=\arg \min \left(\frac{\mathrm{RI}_{\mathrm{ref}}-\|C(\theta)\| \beta_{2}(\theta) \varepsilon}{\|C(\theta)\|\left[1+\beta_{2}(\theta)\right]\left\|A_{2}^{-1}(\theta) B_{\delta}(\theta)\right\|}\right),
$$

then

$$
|\delta|_{\max }(\varepsilon)=\pi\left(\theta_{r 2}, \varepsilon\right)
$$

Notice that there exists a maximum bound for the initial conditions, $\varepsilon$, such that $|\delta|_{\max } \geq 0$ and it is given by

$$
\frac{\mathrm{RI}_{\mathrm{ref}}}{\left\|C\left(\theta_{r 2}\right)\right\| \beta_{2}\left(\theta_{r 2}\right)}=\varepsilon_{\max }
$$

If the initial conditions are at the origin, the maximum perturbation $|\widehat{\delta}|_{\max }$ is

$$
\begin{aligned}
|\widehat{\delta}|_{\max } & \leq \pi\left(\theta_{r 2}, 0\right) \\
& =\frac{\mathrm{RI}_{\mathrm{ref}}}{\left\|C\left(\theta_{r 2}\right)\right\|\left[1+\beta_{2}\left(\theta_{r 2}\right)\right]\left\|A_{2}^{-1}\left(\theta_{r 2}\right) B_{\delta}\left(\theta_{r 2}\right)\right\|} .
\end{aligned}
$$

From expression (23) the maximum bound of perturbation $\delta$ depends on the initial conditions, being maximum at the origin (i.e., $|\widehat{\delta}|_{\max }$ ). Using this fact one can define a switching criterion using the boundary $\left\|x_{0}\right\| \leq \varepsilon^{*} \leq \varepsilon$ as a switching surface, which will be stable for perturbations satisfying $0<\left|\delta^{*}\right|<|\widehat{\delta}|_{\max }$. That is

$$
\varepsilon^{*}=\frac{\mathrm{RI}_{\mathrm{ref}}-\left\|C\left(\theta_{r 2}\right)\right\|\left[1+\beta_{2}\left(\theta_{r 2}\right)\right]\left\|A_{2}^{-1}\left(\theta_{r 2}\right) B_{\delta}(\theta)_{r 2}\right\|\left|\delta^{*}\right|}{\left\|C\left(\theta_{r 2}\right)\right\| \beta_{2}\left(\theta_{r 2}\right)},
$$

where $\delta^{*}<|\widehat{\delta}|_{\max }$ is a design value that is chosen to compute $\varepsilon^{*}$. In other words, the computation of (26) only requires the (nominal) parameters of vertexes $\theta_{r 2}$.

Let the switching criterion from Mode 1 to Mode 2 be as follows:

$$
\text { if }\|x\| \geq \varepsilon^{*} \text { then Mode } 2 \text { is active, }
$$

with $\varepsilon^{*}$ given by (26); then $|\mathrm{RI}(t)| \leq \mathrm{RI}_{\text {ref }}$ for $|\delta| \leq|\widehat{\delta}|_{\max }$.

Until now we have proved that the switching criterion from Mode 1 to Mode 2 keeps the trajectories of the system within $\Omega$. However, to obtain a noninvasive property of the controller, the control must switch back to open-loop conditions as soon as the risk has passed. Since $A_{2}(\theta)$ is Hurwitz and $|\delta| \leq|\widehat{\delta}|_{\max }$, the trajectories of Mode 2 as $t \rightarrow$ $\infty$ will tend to

$$
\lim _{t \rightarrow \infty}\left|\operatorname{RI}(t)_{\text {Mode } 2}\right| \leq\left\|C(\theta) A_{2}^{-1}(\theta) B_{\delta}(\theta)\right\||\hat{\delta}|_{\max }=\Lambda_{2},
$$

while in Mode 1 the norm of the RI tends to

$$
\lim _{t \rightarrow \infty}\left|\mathrm{RI}(t)_{\text {Mode } 1}\right| \leq\left\|C(\theta) A_{1}^{-1}(\theta) B_{\delta}(\theta)\right\||\widehat{\delta}|_{\max }=\Lambda_{1} .
$$

From the controllability property of $(A(\theta), B(\theta))$ there exists a sufficiently large value of $K$, such that $\Lambda_{1}>\Lambda_{2}$ for all $\theta$ in the simplex. Let $\Omega_{\Lambda_{1}} \triangleq\left\{x\left(t_{0}, t, x_{0}, \theta\right)|| \mathrm{RI}(t) \mid<\Lambda_{1}\right\}$ and $\Omega_{\Lambda_{2}} \triangleq\left\{x\left(t_{0}, t, x_{0}, \theta\right)|| R I(t) \mid<\Lambda_{2}\right\}$; then it is satisfied that $\Omega_{\Lambda_{1}} \supset \Omega \supset \Omega_{\Lambda_{2}}$. That $\Omega \supset \Omega_{\Lambda_{2}}$ is a consequence of (25). On the other hand, the maximum perturbation $|\widehat{\delta}|_{\max , \text { la }}$ such that trajectories remain in $\Omega$ at open-loop conditions is

$$
|\delta|_{\mathrm{la}}<\pi_{\mathrm{la}}(\theta, \varepsilon)=\frac{\mathrm{RI}_{\mathrm{ref}}-\|C(\theta)\| \beta_{1}(\theta) \varepsilon}{\|C(\theta)\|\left[1+\beta_{1}(\theta)\right]\left\|A_{1}^{-1}(\theta) B_{\delta}(\theta)\right\|}
$$

with $\left\|e^{A_{1}(\theta) t}\right\| \leq \beta_{1}(\theta) e^{r_{1}(\theta) t}, r_{1}<0$. Let

$$
\theta_{r 1}=\arg \min \left\{\frac{\mathrm{RI}_{\mathrm{ref}}-\|C(\theta)\| \beta_{1}(\theta) \varepsilon}{\|C(\theta)\|\left[1+\beta_{1}(\theta)\right]\left\|A_{1}^{-1}(\theta) B_{\delta}(\theta)\right\|}\right\},
$$

then

$$
|\delta|_{\text {max,la }}(\varepsilon)<\pi_{\mathrm{la}}\left(\theta_{r 1}, \varepsilon\right),
$$

which means that the larger value of the perturbation that can be tolerated is at $\varepsilon=0$; that is,

$$
\begin{aligned}
|\widehat{\delta}|_{\text {max }, \text { la }} & <\pi_{\mathrm{la}}\left(\theta_{r 1}, 0\right) \\
& =\frac{\mathrm{RI}_{\mathrm{ref}}}{\left\|C\left(\theta_{r 1}\right)\right\|\left[1+\beta_{1}\left(\theta_{r 1}\right)\right]\left\|A_{1}^{-1}\left(\theta_{r 1}\right) B_{\delta}(\theta)_{r 1}\right\|} .
\end{aligned}
$$

From (33) and (25) we have that $|\widehat{\delta}|_{\max , \text { la }}<|\widehat{\delta}|_{\max }$, for a sufficient large value of $K$. Therefore if $|\widehat{\delta}|_{\max }$ is applied at Mode 1 the trajectories can be outside $\Omega$. Now, since $A_{i}(\theta)$ are 
Hurwitz, the set $\Omega_{\Lambda_{i}}$ is attractive for the Mode $i$; therefore, any switching surface $\|x\|<\varepsilon^{*}$ with $x \in \Omega$ serves as a switching surface from Mode 2 to Mode 1 to ensure the control objective (13); that is,

$$
\text { if }\|x\|<\varepsilon^{*} \text { then Mode } 1 \text { is active. }
$$

Since the condition to switch from Mode 2 to Mode 1 (34) is arbitrarily smaller than that to switch from Mode 1 to Mode 2 (27), fast switching actions may be present. To avoid this phenomenon a minimum residence time $\left(t_{r}>0\right)$ at Mode 2 can be used to avoid performance degradation. That is, if $|\delta| \leq|\widehat{\delta}|_{\max }$ and the switching criterion is defined as follows:

if $\|x\| \geq \varepsilon^{*}$ then Mode 2 is active

if $\|x\|<\varepsilon^{*}$ and Mode 2 has been active at least a time

$t_{r}>0$ then Mode 1 is active,

where $\varepsilon^{*}$ is given by expression (26), then $|\mathrm{RI}(t)| \leq \mathrm{RI}_{\text {ref }}$, for all time $t>0$.

4.1. Predictive Switching Criterion. The result in the section above establishes sufficient conditions for stability under switching. In the following we will establish a criterion to define the switching surface $\varepsilon^{*}$, which depends on the prediction of the rollover risk, which constitutes a time varying switching surface. Without loss of generality let the present time $t=0$; now let us compute the required initial conditions such that $|\mathrm{RI}(T)|=\mathrm{RI}_{\text {ref }}$; since the rollover prediction risk must be performed in open-loop conditions only Mode 1 is analyzed (see Figure 3):

$$
\begin{aligned}
\left\|\mathrm{RI}_{\mathrm{ref}}\right\|= & \left\|C\left(\theta_{r 1}\right)\right\| \beta_{1}\left(\theta_{r 1}\right) e^{r_{1}\left(\theta_{r 1}\right) T} \widetilde{\mathcal{\varepsilon}} \\
& +\left\|C\left(\theta_{r 1}\right)\right\|\left[1+\beta_{1}\left(\theta_{r 1}\right) e^{r_{1}\left(\theta_{r 1}\right) T}\right] \\
& \times\left\|A_{1}^{-1}\left(\theta_{r 1}\right) B_{\delta}\left(\theta_{r 1}\right)\right\||\delta|,
\end{aligned}
$$

with $|\delta| \in\left[0,|\widehat{\delta}|_{\max }\right]$ hence $\widetilde{\mathcal{\varepsilon}}$ is the value of the switching surface at present time to avoid the imminent risk $\|\mathrm{RI}(T)\|=$ $\left\|\mathrm{RI}_{\text {ref }}\right\|$. Consider

$$
\begin{aligned}
\widetilde{\mathcal{E}}= & \mathrm{RI}_{\mathrm{ref}}-\left\|C\left(\theta_{r 1}\right)\right\|\left[1+\beta_{1}\left(\theta_{r 1}\right) e^{r_{1}\left(\theta_{r 1}\right) T}\right] \\
& \times\left\|A_{1}\left(\theta_{r 1}\right)^{-1} B_{\delta}\left(\theta_{r 1}\right)\right\||\delta| \\
& \times\left(\left\|C\left(\theta_{r 1}\right)\right\| \beta_{1}\left(\theta_{r 1}\right) e^{r_{1}\left(\theta_{r 1}\right) T}\right)^{-1} .
\end{aligned}
$$

Let $|\delta|=|\widehat{\delta}|_{\text {max }}$; hence substituting (25) in (37)

$$
\widetilde{\varepsilon}=\frac{\mathrm{RI}_{\mathrm{ref}}\left[1-Q\left(\theta_{r 1}, \theta_{r 2}\right)\right]}{\left\|C\left(\theta_{r 1}\right)\right\| \beta_{1}\left(\theta_{r 1}\right) e^{r_{1}\left(\theta_{r 1}\right) T}},
$$

with

$$
Q\left(\theta_{r 1}, \theta_{r 1}\right)=\frac{\left[1+\beta_{1}\left(\theta_{r 1}\right) e^{r_{1}\left(\theta_{r 1}\right) T}\right]\left\|A_{1}\left(\theta_{r 1}\right)^{-1} B_{\delta}\left(\theta_{r 1}\right)\right\|}{\left[1+\beta_{2}\left(\theta_{r 2}\right)\right]\left\|A_{2}\left(\theta_{r 2}\right)^{-1} B_{\delta}\left(\theta_{r 2}\right)\right\|} .
$$

TABLE 1: 2000 Cherokee nominal parameters.

\begin{tabular}{lc}
\hline$c$ & $4000 \mathrm{~N} \cdot \mathrm{m} \cdot \mathrm{s} / \mathrm{rad}$ \\
$c_{v}$ & $90240 \mathrm{~N} / \mathrm{rad}$ \\
$c_{h}$ & $180000 \mathrm{~N} / \mathrm{rad}$ \\
$h$ & $0.375 \mathrm{~m}$ \\
$J_{z}$ & $1280 \mathrm{~kg} \cdot \mathrm{m}^{2}$ \\
$J_{x}$ & $362.6 \mathrm{~kg} \cdot \mathrm{m}^{2}$ \\
$k$ & $36075 \mathrm{n} \cdot \mathrm{m} / \mathrm{rad}$ \\
$L_{v}$ & $1.102 \mathrm{~m}$ \\
$L_{h}$ & $1.25 \mathrm{~m}$ \\
$m$ & $1224 \mathrm{~kg}$ \\
$b$ & $1.51 \mathrm{~m}$ \\
\hline
\end{tabular}

Notice that $1-Q\left(\theta_{r 1}, \theta_{r 2}\right)$ can be either positive or negative. Negative values mean that no predicted risk of rollover exists and it is not necessary to switch to Mode 2 at present time. However, as $1-Q\left(\theta_{r 1}, \theta_{r 2}\right)$ becomes positive, the switching is possible with

$$
\widetilde{\varepsilon} \geq \varepsilon_{\max }
$$

with $\varepsilon_{\max }$ given by (24). From this point, stability can be derived as in the section above using $\varepsilon^{*}=\widetilde{\varepsilon}$.

\section{Numerical Simulations}

In this section, numerical evaluations of the proposed control strategy are used to illustrate the controller performance. Firstly, a numerical sensitivity analysis is performed to determine the vertexes of the polytope corresponding to (10). Afterward, the evaluation of the proposed controller using CarSim and three different vehicles is performed.

\subsection{Numerical Sensitivity Analysis and Vertexes Computation.} In order to keep to a minimum the number of the vertexes of the polytopic matrix, it is convenient to determine which parameters are more significant in the system description. To this end, a sensitivity study is performed. One of the simplest methods to determine parameter sensitivity is to compute the output response for a variety of maneuvers, in face of parameter variations. The parameters are perturbed using fixed steps of 5\%, from a lower to an upper bound. The sensitivity index (SI) is defined as [28]

$$
\mathrm{SI}=\frac{\widehat{y}_{\max }-\widehat{y}_{\min }}{\widehat{y}_{\max }} \cdot 100,
$$

where $y$ is the measured variable, $\widehat{y}_{\max }=\max \left\{y_{\max }\left(\varrho_{\text {lower }}\right)\right.$, $\left.y_{\max }\left(\varrho_{\text {lower }}+0.05 \varrho_{\text {lower }}\right), y_{\max }\left(\varrho_{\text {lower }}+0.1 \varrho_{\text {lower }}\right), \ldots, y\left(\varrho_{\text {upper }}\right)\right\}$, where $\varrho_{\text {lower }}$ and $\varrho_{\text {upper }}$ are the lower and upper bound of the parameters. On the other hand, $\widehat{y}_{\min }=$ $\min \left\{y_{\max }\left(\varrho_{\text {lower }}\right), y_{\max }\left(\varrho_{\text {lower }}+0.05 \varrho_{\text {lower }}\right), y_{\max }\left(\varrho_{\text {lower }}+\right.\right.$ $\left.\left.0.1 \varrho_{\text {lower }}\right), \ldots, y\left(\varrho_{\text {upper }}\right)\right\}$. In this paper, $y$ is the rollover index.

Nominal parameters are displayed in Table 1, while Table 2 shows the maximum SI values of the each parameter. It can be observed that the vehicle dynamics are very sensitive to the design dependent values $c_{v}$ (related with the tire), 
TABLE 2: Sensitivity index.

\begin{tabular}{lc}
\hline Parameter & SI \\
\hline$c_{v}$ & $11.65 \%$ \\
$h$ & $10.88 \%$ \\
$L_{h}$ & $9.01 \%$ \\
$L_{v}$ & $6.84 \%$ \\
$v_{\mathrm{CoG}}$ & $6.76 \%$ \\
$m$ & $5.37 \%$ \\
$c_{h}$ & $5.06 \%$ \\
$c$ & $1.83 \%$ \\
$J_{x}$ & $1.12 \%$ \\
$k$ & $0.62 \%$ \\
$J_{z}$ & $0.2 \%$ \\
\hline
\end{tabular}

$h$ (distance of the CoG from the road), $L_{v}$ and $L_{h}$ (distance from CoG to the front, rear axles resp.), and the vehicle speed $v_{\mathrm{CoG}}$. Let us fix $L_{h}$ and $L_{v}$ at their nominal values since their variation is usually small; then the vertexes of the matrices are defined with respect to the parameters $c_{v}, h$, and $v_{\mathrm{CoG}}$ for simplicity. $A(\theta)$ and $B_{\delta}(\theta)$ can be expressed as a convex function of the time-varying parameter vector:

$$
\theta=\left[\theta_{1}, \theta_{2}, \theta_{3}, \theta_{4}, \theta_{5}, \theta_{6}, \theta_{7}, \theta_{8}\right]
$$

as described as follows:

$$
\begin{gathered}
A(\theta)=\sum_{i=1}^{8} \theta_{i} A_{i}, \\
B_{\delta}(\theta)=\sum_{i=1}^{8} \theta_{i} B_{\delta_{i}},
\end{gathered}
$$

where

$$
\begin{aligned}
A_{1}=A\left(\underline{c_{v}}, \underline{h}, \underline{v_{\mathrm{CoG}}}\right), & A_{2}=A\left(\underline{c_{v}}, \underline{h}, \overline{v_{\mathrm{CoG}}}\right), \\
A_{3}=A\left(\underline{c_{v}}, \bar{h}, \underline{v_{\mathrm{CoG}}}\right), & A_{4}=A\left(\underline{c_{v}}, \bar{h}, \overline{v_{\mathrm{CoG}}}\right), \\
A_{5}=A\left(\overline{c_{v}}, \underline{h}, \underline{v_{\mathrm{CoG}}}\right), & A_{6}=A\left(\overline{c_{v}}, \underline{h}, \overline{v_{\mathrm{CoG}}}\right), \\
A_{7}=A\left(\overline{c_{v}}, \bar{h}, \underline{v_{\mathrm{CoG}}}\right), & A_{8}=A\left(\overline{c_{v}}, \bar{h}, \overline{v_{\mathrm{CoG}}}\right), \\
B_{\delta_{1}}=B_{\delta}\left(\underline{c_{v}}, \underline{h}, \underline{v_{\mathrm{CoG}}}\right), & B_{\delta_{2}}=B_{\delta}\left(\underline{c_{v}}, \underline{h}, \overline{v_{\mathrm{CoG}}}\right), \\
B_{\delta_{3}}=B_{\delta}\left(\underline{c_{v}}, \bar{h}, \underline{v_{\mathrm{CoG}}}\right), & B_{\delta_{4}}=B_{\delta}\left(\underline{c_{v}}, \bar{h}, \overline{v_{\mathrm{CoG}}}\right), \\
B_{\delta_{5}}=B_{\delta}\left(\overline{c_{v}}, \underline{h}, \underline{v_{\mathrm{CoG}}}\right), & B_{\delta_{6}}=B_{\delta}\left(\overline{c_{v}}, \underline{h}, \overline{v_{\mathrm{CoG}}}\right), \\
B_{\delta_{7}}=B_{\delta}\left(\overline{c_{v}}, \bar{h}, \underline{v_{\mathrm{CoG}}}\right), & B_{\delta_{8}}=B_{\delta}\left(\overline{c_{v}}, \bar{h}, \overline{v_{\mathrm{CoG}}}\right) .
\end{aligned}
$$

In this paper $v_{\mathrm{CoG}}=25 \mathrm{~m} / \mathrm{s}=90 \mathrm{Km} / \mathrm{h}$, and $\overline{v_{\mathrm{CoG}}}=$ $40 \mathrm{~m} / \mathrm{s}=\overline{144} \mathrm{Km} / \mathrm{h}$. A controller gain vector that guarantees stability for all vertexes is $\mathrm{K}=$ mg $\left[\begin{array}{lllll}-7.1287 & 0.9842 & 0.3271 & -0.0944\end{array}\right]$.

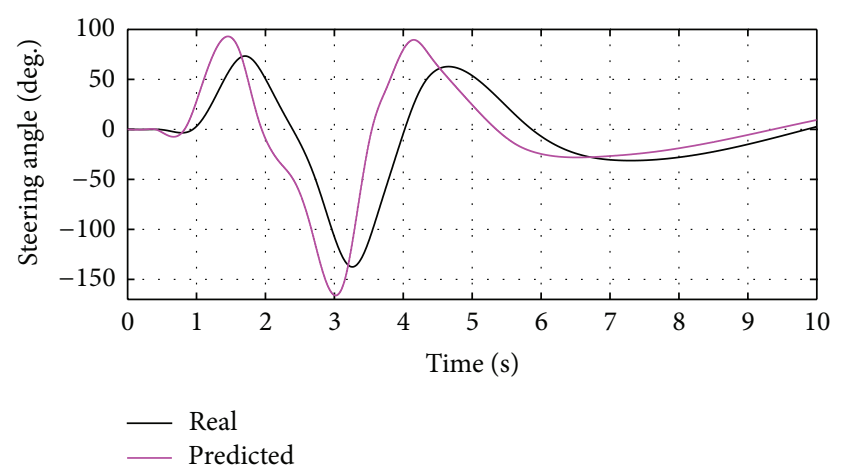

FIGURE 4: Steering wheel angle real versus predicted.

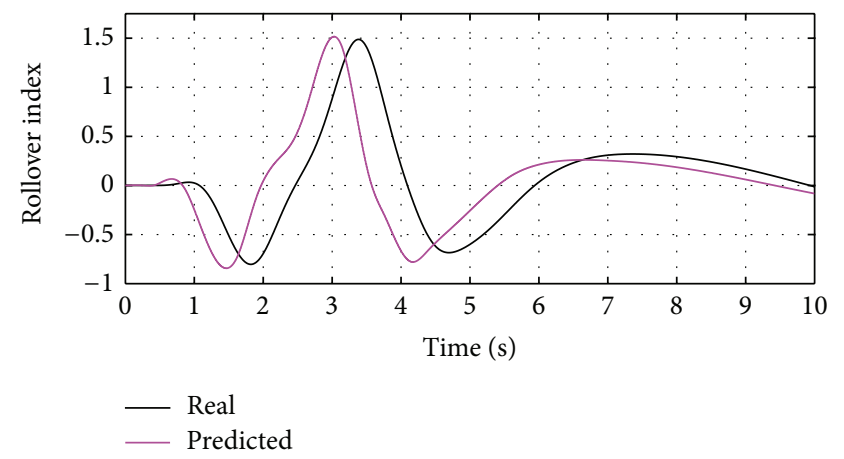

FIGURE 5: Rollover index real versus predicted (open loop).

5.2. The Predictive Controller. The first step to implement the predictive controller in Figures 2 and 3 is to predict the front wheels angle $(\delta)$ given by the driver's behavior. In this work a zero-order model is used to perform the prediction as in [27]. Afterward, the RI at open-loop conditions is computed along a given prediction horizon. The results are displayed in Figures 4 and 5 for a double lane-change maneuver with rollover risk.

Secondly the predicted RI is used to perform the switching, using the algorithm in Figure 3. Two double lane-change maneuvers are chosen to illustrate the performance of the switched controller: (i) a first one with rollover risk along the $[3,3.8]$ seconds interval in open loop (see Figure 6) and (ii) a second one without rollover risk (see Figure 7).

The comparison of the proposed strategy with respect to a robust nonswitched linear action and open loop dynamics is performed. The proposed controller is able to prevent rollover and to maintain the RI within the specified values. Moreover, the control action is reduced significantly with respect to the nonswitched alternative and it is used only when necessary to avoid rollover (in Figure 7 the braking control actions are not performed).

The effect of the prediction horizon is evaluated in the proposed scheme for a given maximum value of $|\delta|<$ $|\widehat{\delta}|_{\max }$; the results are displayed in Figure 8. As the prediction horizon is increased the controller becomes more invasive; however the actual values of RI are smaller. In every case, RI is kept within the required values. 


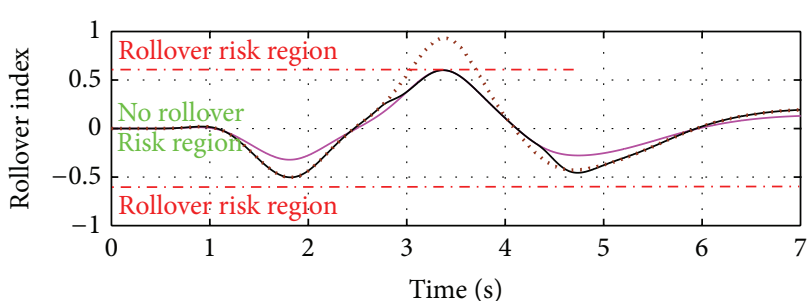

…... Open loop

— Robust nonswitched

- Switched robust predictive

(a)

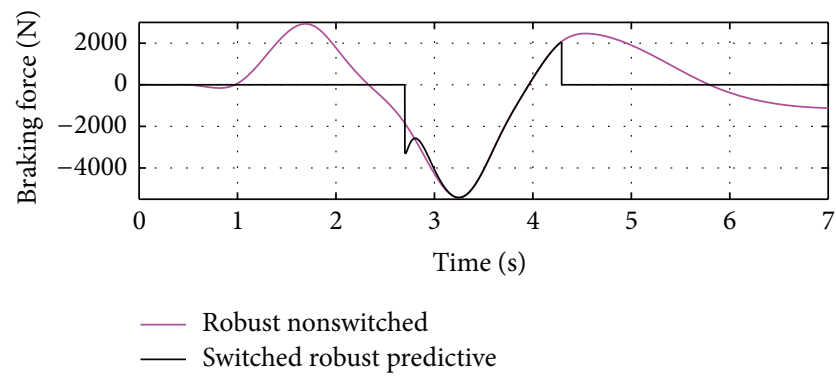

(b)

FIgURE 6: Rollover index and braking force evolution for a double lane-change maneuver with rollover risk.

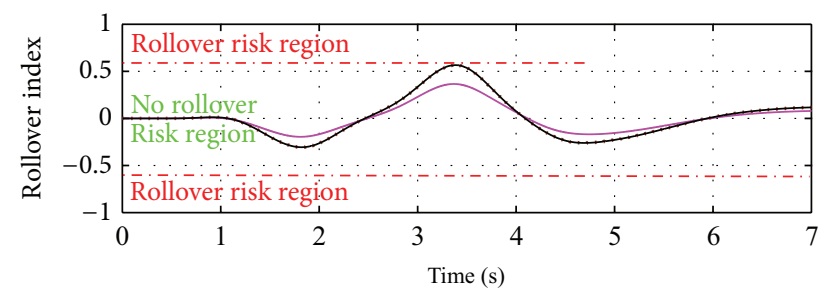

...... Open loop

— Robust nonswitched

— Switched robust predictive

(a)

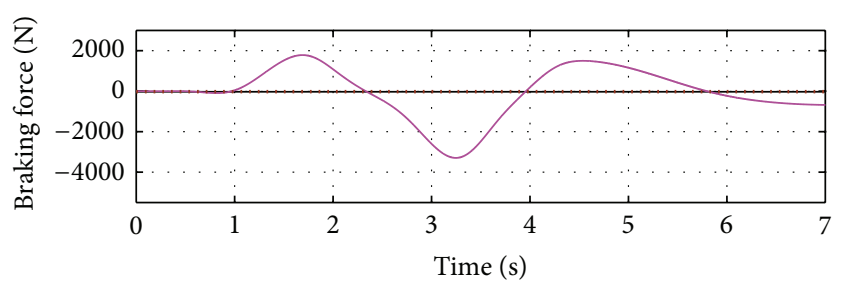

..... Open loop

— Robust nonswitched

— Switched robust predictive

(b)

FIGURE 7: Rollover index and braking force evolution for a double lane-change maneuver without rollover risk.

To illustrate the robustness of the proposed scheme, the controller is evaluated using models from CarSim of three vehicles: a 2000 Porsche 911 sport car, a 2002 Mitsubishi Mini U62T utility truck, and a Jeep Cherokee 2000 SUV. The nominal parameters for these vehicles are shown in Table 3.

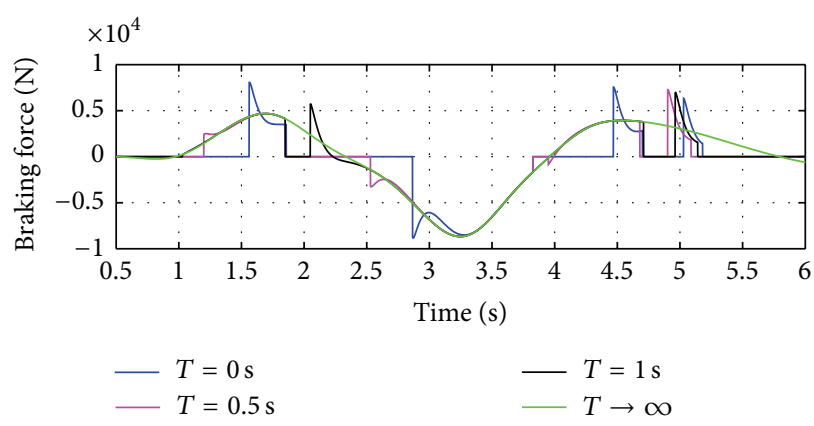

(a)

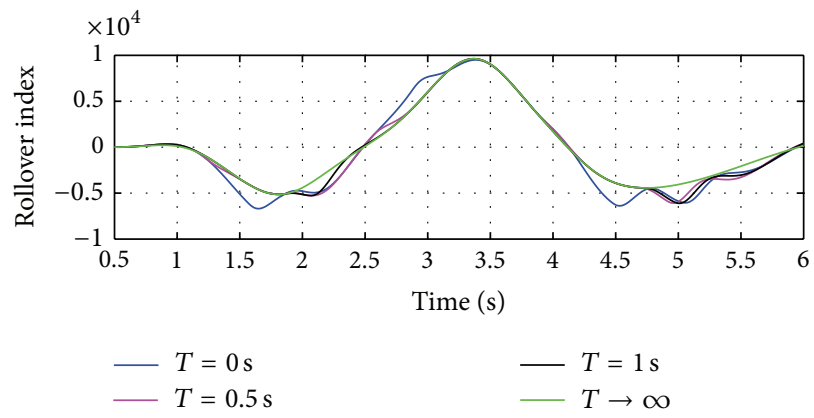

(b)

FIGURE 8: Effect of prediction horizon on system performance.

TABle 3: Nominal parameters.

\begin{tabular}{lcccc}
\hline Value & Cherokee & Porsche & U62T & Units \\
\hline$h$ & 0.375 & 0.010 & 0.200 & $\mathrm{~m}$ \\
$J_{z}$ & 1280 & 1270 & 686 & $\mathrm{~kg} \cdot \mathrm{m}^{2}$ \\
$J_{x}$ & 362.6 & 614 & 384 & $\mathrm{~kg} \cdot \mathrm{m}^{2}$ \\
$L_{v}$ & 1.102 & 1.525 & 0.550 & $\mathrm{~m}$ \\
$L_{h}$ & 1.25 & 0.825 & 1.373 & $\mathrm{~m}$ \\
$m$ & 1224 & 1278 & 600 & $\mathrm{~kg}$ \\
$b$ & 1.51 & 1.72 & 1.26 & $\mathrm{~m}$ \\
\hline
\end{tabular}

An aggressive double lane-change is performed at $120 \mathrm{Km} / \mathrm{h}$ and the open loop behaviors of the three vehicles are shown in Figure 9. Since the Porsche has a smaller $h$ (among other differences), the maneuver does not lead to rollover but to lateral slip, while for the Cherokee and the $\mathrm{U} 62 \mathrm{~T}$ the rollover is present. In Figure 10 the proposed controller is applied to the three cars. In this case, the controller that uses the model of the Jeep Cherokee is used also for the Porsche and the utility truck. The controller is able to prevent rollover in the Jeep Cherokee, and surprisingly the same controller is able to prevent rollover in the case of the truck, even if considerable differences exist. As can be observed the controller leads to a further decrement on the velocity to compensate for the higher CoG, among other differences. On the other hand, it is also surprising that the skid and $180^{\circ}$ spin of the sport vehicle can be prevented. 


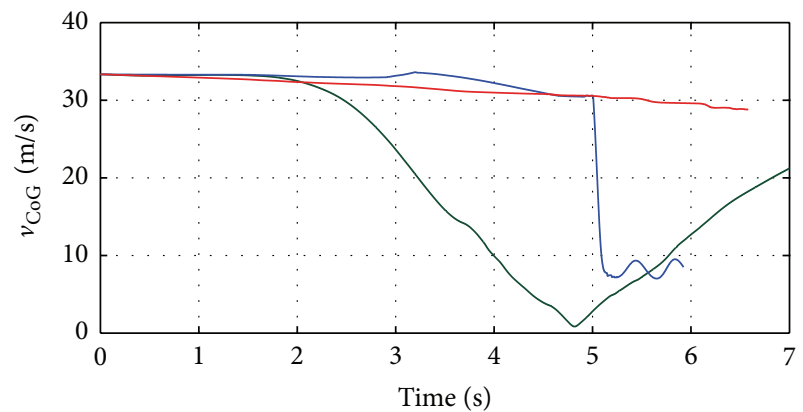

(a)

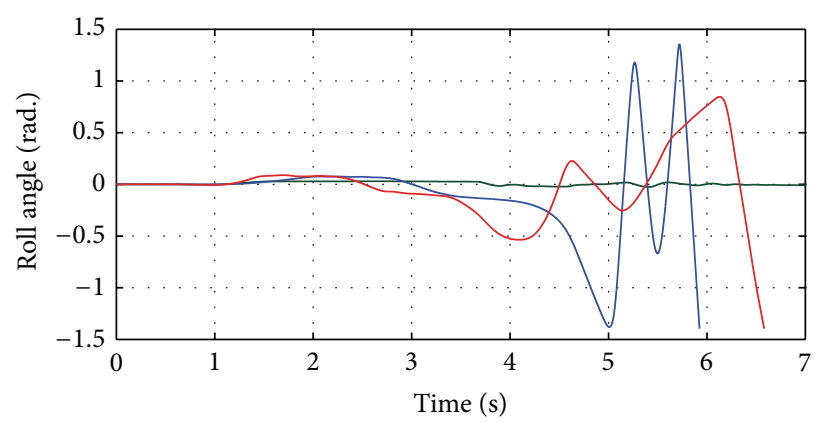

- Porsche

- U62T

— Cherokee

(c)

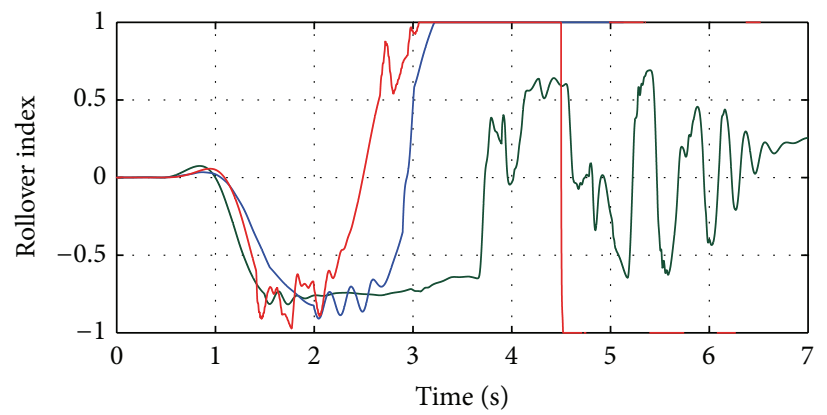

(b)

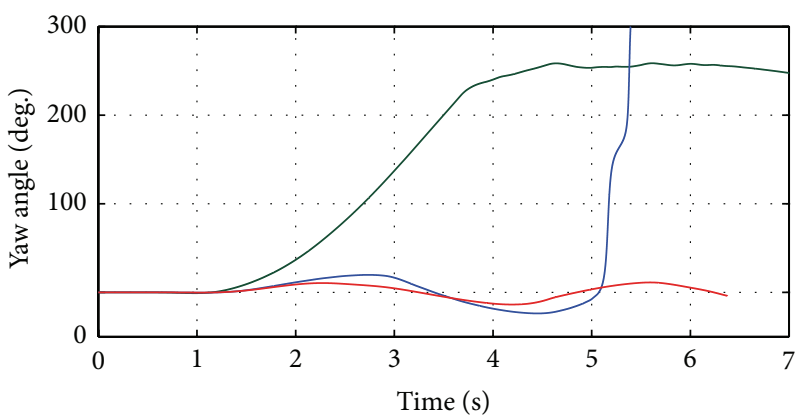

Porsche
U U62T

— Cherokee

FIGURE 9: Dynamic response comparative in open loop for an aggressive double lane-change maneuver.

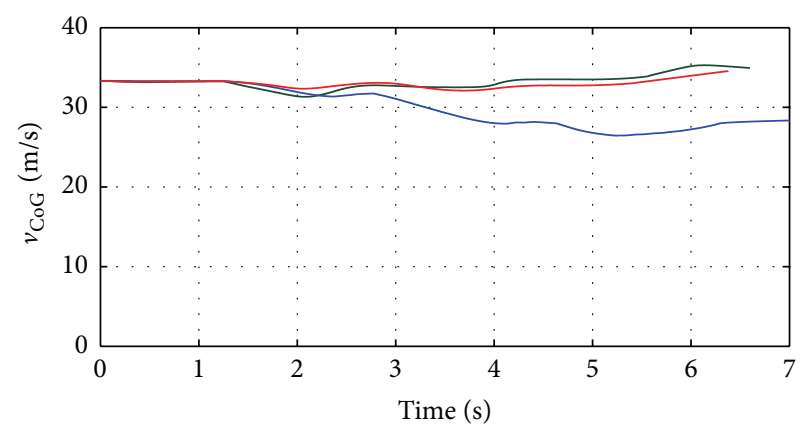

(a)

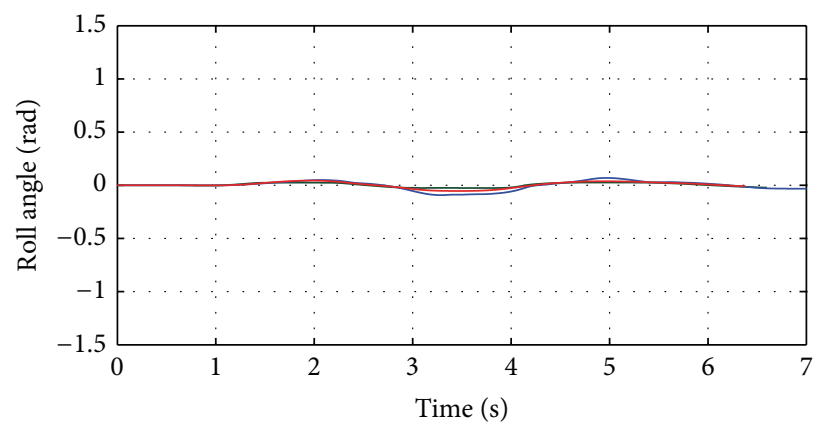

Porsche
U62T
Cherokee

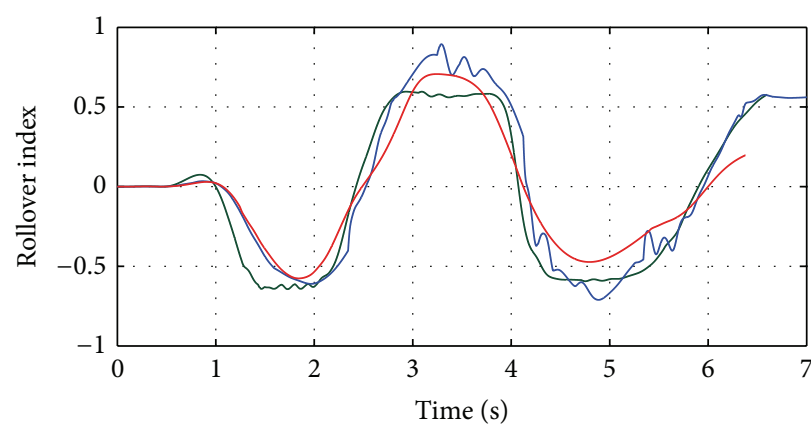

(b)

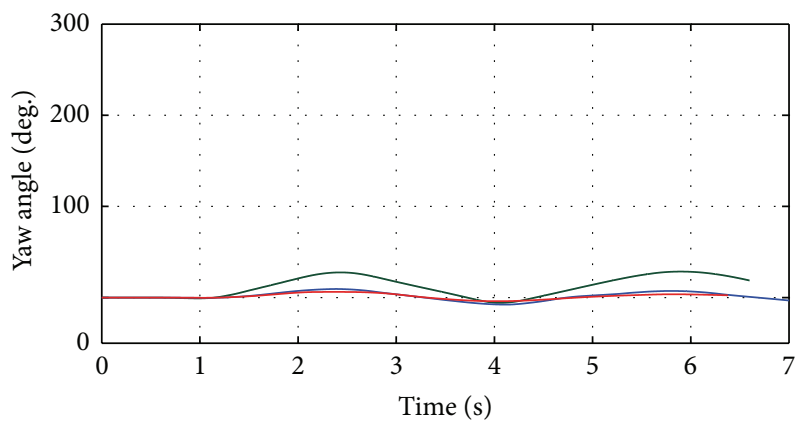

— Porsche

- U62T

— Cherokee

(c)

(d)

FIgURE 10: Dynamic response comparative with the proposed controller active for an aggressive double lane-change maneuver. 


\section{Conclusions}

In this paper a robust switched controller with a commutation dependent on the RI prediction is proposed. The stability of the controller is derived in two steps. Firstly, by noticing that a state-dependent switching criterion ensures the invariance of a suitable set $\Omega$ for a given $|\widehat{\delta}|_{\max }$, and secondly using RI prediction to define a time-varying state-dependent switching criterion. The results are illustrated with simulations and the robustness of the proposed scheme is verified for different vehicle models. The proposed controller is robust and it has displayed an unexpected capacity of preventing skidding; this last feature must be further analyzed.

\section{Nomenclature}

$\begin{array}{ll}\alpha_{v}, \alpha_{h}: & \text { Front, rear tire side slip angle } \\ \beta: & \text { Vehicle side slip angle } \\ \delta: & \text { Front wheels angle } \\ \psi: & \text { Vehicle yaw angle } \\ \varphi: & \text { Chassis roll angle } \\ b: & \text { Track width } \\ c: & \text { Damping coefficient } \\ c_{v}, c_{h}: & \text { Front, rear tire stiffness coefficient } \\ F_{\mathrm{ZL}}, F_{\mathrm{ZR}}: & \text { Left, right contact point vertical forces } \\ g: & \text { Gravity } \\ h: & \text { Distance of CoG from the road } \\ J_{z}, J_{x}: & \text { Moment of inertia about the vertical, } \\ & \text { longitudinal axis } \\ k: & \text { Spring stiffness coefficient of the } \\ L_{v}, L_{h}: & \text { suspension } \\ m: & \text { Distance from CoG to the front, rear axle } \\ \mathrm{RI}: & \text { Rehicle mass } \\ T: & \text { Pollover index } \\ u: & \text { Prediction horizon } \\ u_{\mathrm{max}}: & \text { Differential braking force } \\ v_{\mathrm{CoG}}: & \text { Maximum differential braking force } \\ & \text { inertial coordinate system as seen in the } \\ & \text { vehicle. }\end{array}$

\section{Conflict of Interests}

The authors declare that there is no conflict of interests regarding the publication of this paper.

\section{Acknowledgments}

The authors acknowledge financial support from CONACYT GRANTS: SENER 152485 and FORDECYT 190966. The authors also acknowledge technical support from Irwin A. Díaz-Diaz.

\section{References}

[1] North American Free Trade Agreement, Land Transportation Standards Subcommittee, "Highway safety performance criteria in support of vehicle weight and dimension regulations: candidate criteria \& recommended thresholds," Working Draft for Consultation, 1999.
[2] S. Takano and M. Nagai, "Dynamics control of large vehicles for rollover prevention," in Proceedings of the Vehicle Electronics Conference, pp. 85-89, Tottori, Japan, 2001.

[3] M. H. Kim, J. H. Oh, J. H. Lee, and M. C. Jeon, "Development of rollover criteria based on simple physical model of rollover event," International Journal of Automotive Technology, vol. 7, no. 1, pp. 51-59, 2006.

[4] X. Wu, X. Ge, and H. Huang, "Study on vehicle rollover avoidance," in Proceedings of the International Conference on Measuring Technology and Mechatronics Automation (ICMTMA '10), pp. 681-684, Changsha, China, March 2010.

[5] G. Phanomchoeng and R. Rajamani, "New rollover index for the detection of tripped and untripped rollovers," IEEE Transactions on Industrial Electronics, vol. 60, no. 10, 2013.

[6] R. Ghandour, A. Victorino, A. Charara, and D. Lechner, "Risk indicators anticipation based on the vehicle dynamics anticipation to avoid accidents," in Proceedings of the IEEE Intelligent Vehicles Symposium, pp. 93-98, Alcala de Henares, España, 2012.

[7] C. Zong, Z. Yu, Q. Miao, and B. Zhang, "Research on rollover warning algorithm of heavy commercial vehicle based on prediction model," in Proceedings of the International Conference on Computer, Mechatronics, Control and Electronic Engineering (CMCE '10), pp. 432-435, Changchun, China, August 2010.

[8] T. Zhu and C. Zong, "An advanced methodology for rollover warning of heavy duty truck based on Kalman filter state estimation," in Proceedings of the International Conference on Intelligent Computation Technology and Automation (ICICTA '10), pp. 466-469, Changsha, China, May 2010.

[9] H. Imine, L. M. Fridman, and T. Madani, "Steering control for rollover avoidance of heavy vehicles," IEEE Transactions on Vehicular Technology, vol. 61, no. 8, pp. 3499-3509, 2012.

[10] J. Kang, J. Yoo, and K. Yi, "Driving control algorithm for maneuverability, lateral stability, and rollover prevention of 4WD electric vehicles with independently driven front and rear wheels," IEEE Transactions on Vehicular Technology, vol. 60, no. 7, pp. 2987-3001, 2011.

[11] M. Islam and C. Ha, "Road vehicle rollover avoidance using active steering controller," in Proceedings of the 14th International Conference on Computer and Information Technology (ICCIT '11), pp. 298-302, Dhaka, Bangladesh, December 2011.

[12] S. Yim, "Design of a preview controller for vehicle rollover prevention," IEEE Transactions on Vehicular Technology, vol. 60, no. 9, pp. 4217-4226, 2011.

[13] S. Solmaz, "Switched stable control design methodology applied to vehicle rollover prevention based on switched suspension settings," IET Control Theory \& Applications, vol. 5, no. 9, pp. 1104-1112, 2011.

[14] H. Horisberger and P. R. Belanger, "Regulators for linear, time invariant plants with uncertain parameters," IEEE Transactions on Automatic Control, vol. 21, no. 5, pp. 705-708, 1976.

[15] G. Chesi, A. Garulli, A. Tesi, and A. Vicino, "Robust stability of time-varying polytopic systems via parameter-dependent homogeneous Lyapunov functions," Automatica, vol. 43, no. 2, pp. 309-316, 2007.

[16] A. BenAbdallah, M. A. Hammami, and J. Kallel, "Robust stability of uncertain piecewise-linear systems: LMI approach," Nonlinear Dynamics, vol. 63, no. 1-2, pp. 183-192, 2011.

[17] S. Solmaz, M. Corless, and R. Shorten, "A methodology for the design of robust rollover prevention controllers for automotive vehicles: part 1-differential braking," in Proceedings of the 45th IEEE Conference on Decision and Control (CDC '06), pp. 17391744, San Diego, Calif, USA, December 2006. 
[18] T. Zhu, B. Wu, and C. Zong, "The design of rollover prevention system in heavy vehicles based on an robust method," in Proceedings of the 2nd Conference on Power Electronics and Intelligent Transportation System (PEITS '09), pp. 98-101, Shenzhen, China, December 2009.

[19] M. Akar and A. D. Dere, "A switching rollover controller coupled with closed-loop adaptive vehicle parameter identification," IEEE Transactions on Intelligent Transportation Systems, no. 99, pp. 1-7, 2014.

[20] C. Larish, D. Piyabongkarn, V. Tsourapas, and R. Rajamani, “A new predictive lateral load transfer ratio for rollover prevention systems," IEEE Transactions on Vehicular Technology, vol. 62, no. 7, pp. 2928-2936, 2013.

[21] J. Pathompong and O. Toshiyuki, "Stabilization of vehicle rollover by nonlinear model predictive control," in Proceedings of the SICE Annual Conference, pp. 1568-1573, Nagoya, Japan, 2013.

[22] N. Bouton, R. Lenain, B. Thuilot, and P. Martinet, "An active anti-rollover device based on predictive functional control: application to an all-terrain vehicle," in Proceedings of the IEEE International Conference on Robotics and Automation (ICRA '09), pp. 1309-1314, Kobe, Japan, May 2009.

[23] H. B. Pacejka, "Vehicle modeling," in Tyre and Vehicle Dynamics, chapter 4, p. 150, Butterworth-Heinemann, Oxford, UK, 3rd edition, 2012.

[24] U. Kiencke and L. Nielsen, "Vehicle modeling," in Automotive Control Systems for Engine Driveline and Vehicle, chapter 8, p. 360, Springer, San Diego, Calif, USA, 2nd edition, 2005.

[25] S. Solmaz, Topics in automotive rollover prevention: robust and adaptive switching strategies for estimation and control [Ph.D. dissertation], Hamilton Institute, Kildare, Ireland, 2007.

[26] T. Pancake, M. Corless, and M. Brockman, "Analysis and control of polytopic uncertain/nonlinear systems in the presence of bounded disturbance inputs," in Proceedings of the American Control Conference, pp. 159-163, Chicago, Ill, USA, June 2000.

[27] M. R. Licea and I. Cervantes, "On the predictive rollover detection in wheeled vehicles," in Proceedings of the 38th Annual Conference on IEEE Industrial Electronics Society, pp. 2410-2415, Montreal, Canada, 2012.

[28] H. Gardner, "Evaluation of uncertainties in environmental radiological assessment models," Tech. Rep. NUREG/CR-3332, U.S. Nuclear Regulatory Commission, Washington, DC, USA, 1983. 


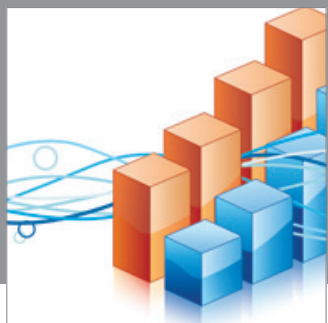

Advances in

Operations Research

mansans

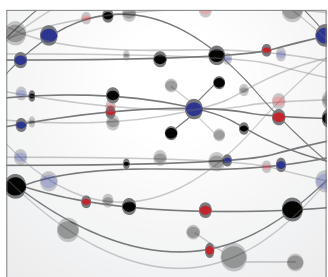

The Scientific World Journal
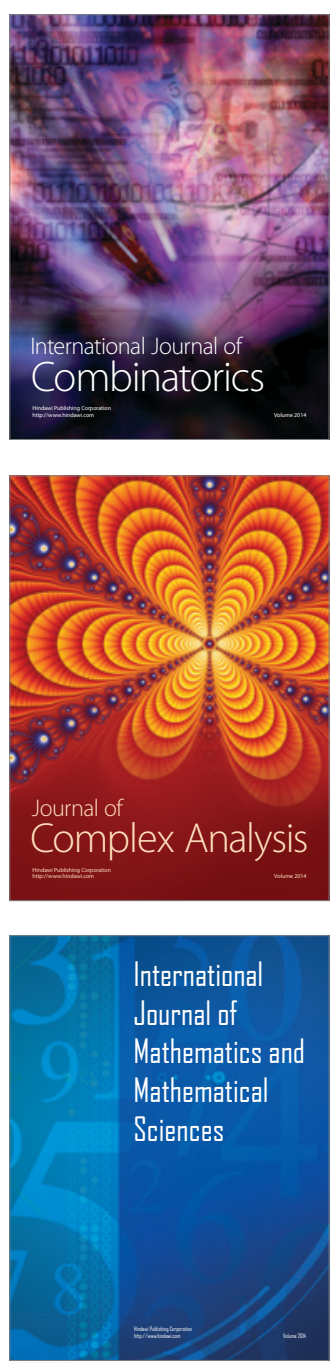
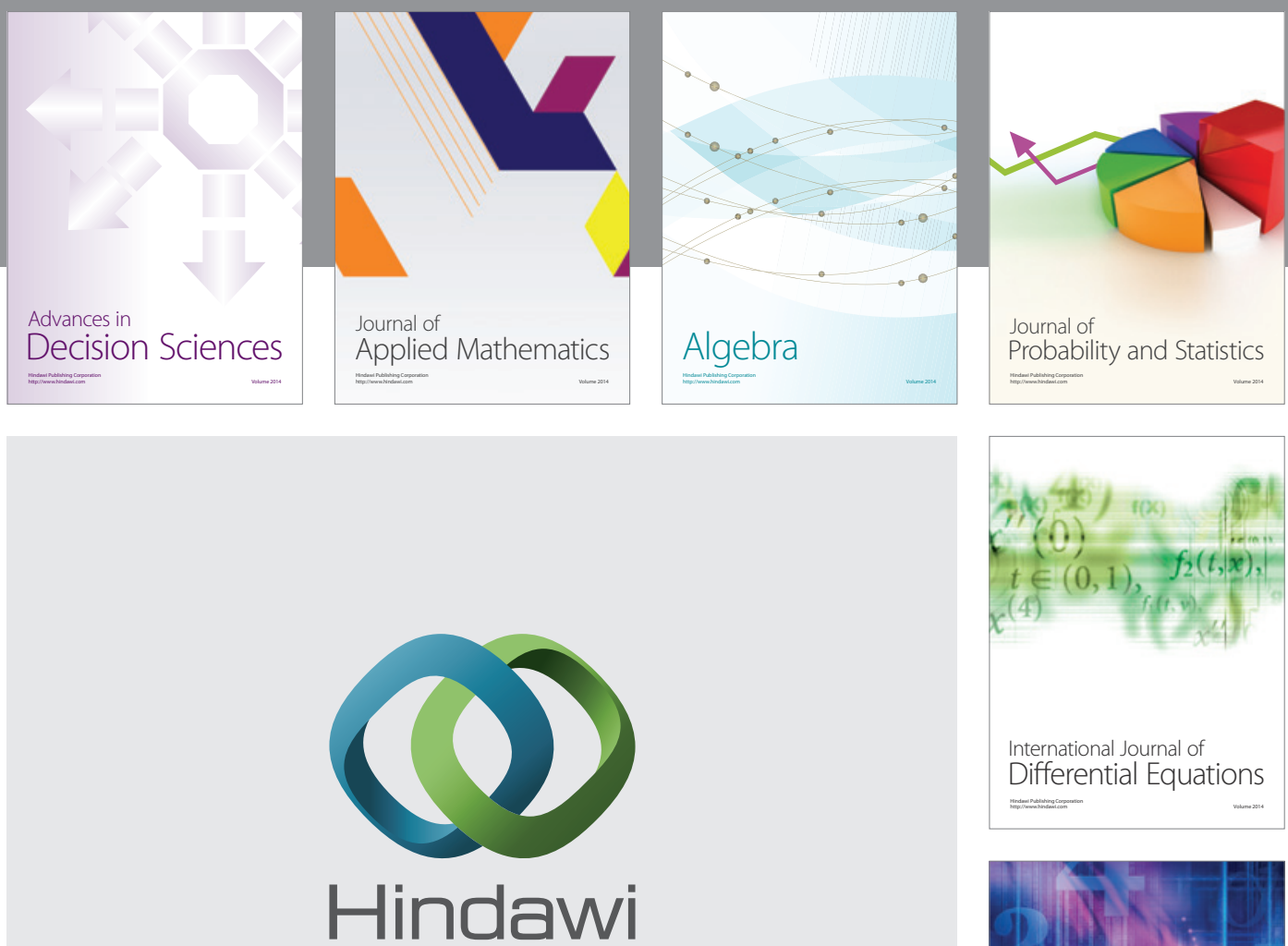

Submit your manuscripts at http://www.hindawi.com
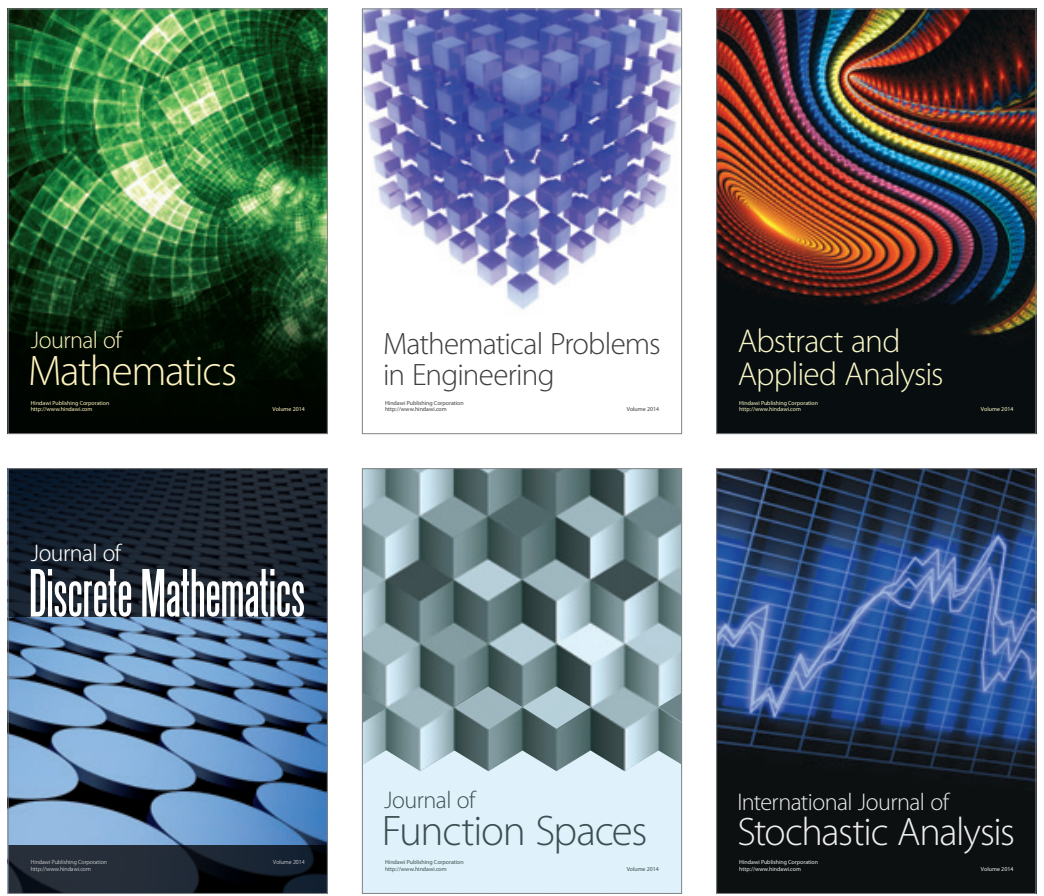

Journal of

Function Spaces

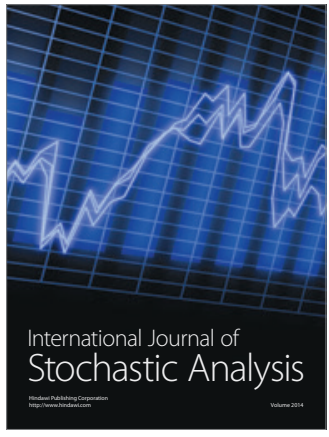

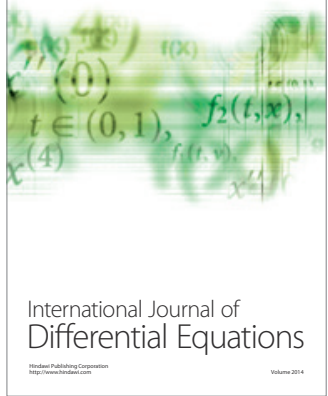
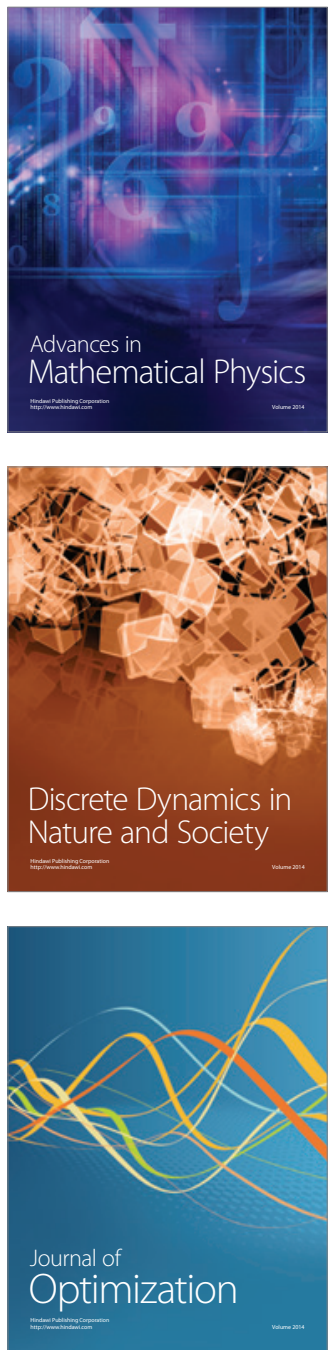